\title{
A Case Report: Regarding Transduodenal Sphincteroplasty
}

\author{
Maria S Ordonez ${ }^{1}$, Jorge A Arteaga ${ }^{2}$, Amber N Himmler ${ }^{3}$, Jeovanni Reinoso ${ }^{4}$, Juan C Salamea ${ }^{5}$
}

\begin{abstract}
Introduction: Stenosing papillitis is rare. It can be primary or secondary and occurs due to the presence of stones in the common bile duct. The initial treatment of choledocholithiasis is endoscopic retrograde cholangiopancreatography (ERCP). However, when ERCP is unsuccessful, the transduodenal sphincteroplasty is an alternative strategy that obtains satisfactory results in select patients.

Case description: A 27-year-old female patient presents right-upper quadrant pain associated with scleral icterus. She had choledocholithiasis and cholestasis. The ERCP performed was failed, so an open cholecystectomy with common bile duct exploration was undertaken. As the ampulla could not be cannulated, a "T" tube was placed. On a postoperative day 15, a cholangiography was performed without evidence of contrast passage into the duodenum. A re-exploration of the bile duct was undertaken 30 days after and a papillary exploration through a transduodenal approach was performed. A new cholangiography was done on postoperative day 9 , at which point adequate passage of contrast into the duodenum was appreciated.
\end{abstract}

Conclusion:Transduodenal sphincteroplasty is a procedure rarely performed in the era of endoscopy. Nonetheless, when these cases do come up, knowing the appropriate surgical technique is crucial to obtain satisfactory results for the patient.

Keywords: Choledocholithiasis, Sphincteroplasty, Transduodenal.

Panamerican Journal of Trauma, Critical Care \& Emergency Surgery (2021): 10.5005/jp-journals-10030-1316

\section{BACKGROUND}

The inflammatory process of the sphincter of Oddi, also known as stenosing papillitis, is generally rare. Stenosing papillitis can be primary or secondary due to the presence of stones in the common bile duct and can cover just portions or the entire sphincteric apparatus. $^{1-3}$

The initial treatment of choledocholithiasis is endoscopic retrograde cholangiopancreatography (ERCP). However, this technique can fail in $0.6-1 \%$ of patients. For this reason, is important to know alternative treatments such as laparoscopic or conventional bile duct exploration, transduodenal sphincterotomy, or even a choledochoduodenostomy. ${ }^{4}$

This review aims to identify and describe transduodenal sphincteroplasty as an alternative procedure for the management of choledocholithiasis. Though rarely performed in the era of endoscopy, it allows for satisfactory results to be obtained in selected patients.

\section{Case Description}

A 27-year-old female patient with no pertinent past medical history presented with 48 hours of colicky right-upper quadrant pain which did not subside with the administration of analgesics. The patient reported that in the 6 hours before the presentation, the abdominal pain had increased in intensity and was newly associated with scleral icterus. In the emergency department, the patient underwent a detailed physical examination, which revealed pain on deep palpation in the right-upper quadrant, but a negative Murphy's sign. Laboratory results demonstrated the absence of leukocytosis or neutrophilia, a normal coagulation profile, and normal renal and pancreatic function. There was laboratory evidence of cholestasis with hyperbilirubinemia $(5.5 \mathrm{mg} / \mathrm{dL}$ ) due to an elevation of direct bilirubin ( $5 \mathrm{mg} / \mathrm{dL}$ ), elevated gamma-glutamyl transferase ( $746 \mathrm{mg} /$ $\mathrm{dL})$, elevated alkaline phosphatase (305 $\mathrm{mg} / \mathrm{dL})$, and transaminitis (AST $660 \mathrm{mg} / \mathrm{dL}$, ALT $520 \mathrm{mg} / \mathrm{dL}$ ). A right-upper quadrant abdominal ultrasound revealed a thin-walled gallbladder with biliary sludge
${ }^{1}$ Department of Surgery, Universidad de Cuenca, Cuenca, Azuay, EC, Cuenca, Ecuador

${ }^{2}$ Department of Surgery, Hospital Vicente Corral Moscoso, Cuenca, Ecuador

${ }^{3}$ Department of Surgery, Medstar Georgetowan University Hospital and Washington Hospital Center, MedStar Georgetown University Hospital, Washington, DC, USA

${ }^{4}$ Department of Cirugía Trauma y Emergencias, Hospital Vicente Corral Moscoso, Cuenca, Ecuador

${ }^{5}$ Department of Surgery, Hospital Universitario Del Rio, Cuenca, Azuay, Ecuador

Corresponding Author: Maria S Ordonez, Department of Surgery, Universidad de Cuenca, Cuenca, Azuay, EC, Cuenca, Ecuador, Phone: +593989892931, e-mail: msoledad_ordonez@hotmail.com

How to cite this article: Ordonez MS, Arteaga JA, Himmler AN, et al. A Case Report: Regarding Transduodenal Sphincteroplasty. Panam J Trauma Crit Care Emerg Surg 2021;10(1):39-42.

Source of support: Nil

Conflict of interest: None

in addition to the presence of a 5- and 7-mm gallstone within the common bile duct. As such, ERCP was attempted. However, the duct could not be cannulated.

After the attempted ERCP, the patient developed mild acute pancreatitis. Forty-eight hours after ERCP, the patient was found to have evidence of cholangitis due to choledocholithiasis with an elevation of bilirubin (total bilirubin of $14.2 \mathrm{mg} / \mathrm{dL}$ and direct bilirubin of $12.5 \mathrm{mg} / \mathrm{dL}$ ), and a new leukocytosis with associated neutrophilia. An open cholecystectomy with the common bile duct was undertaken through a Kocher incision. During this intervention, a thin-walled gallbladder containing multiple small gallstones was extracted. A 4-mm longitudinal incision was made along the dilated common bile duct without removing stones or crossing the sphincter into the duodenum. Manipulating the common bile duct 
and duodenum was technically difficult due to extensive edema and inflammation. A latex " $T$ " tube was inserted into the bile duct and a Jackson Pratt drain was placed in Morrison's pouch. After 3 days, the Jackson Pratt drain was found to have minimal output and was subsequently removed. The " $T$ " tube, however, was kept in place and was found to have an average daily output of $600 \mathrm{~mL}$. The patient's total and direct bilirubin down trended to 4.2 and 3.8 $\mathrm{mg} / \mathrm{dL}$, respectively. As such, the patient was discharged with a plan to reassess as an outpatient after the inflammation had subsided.

On a postoperative day 15 , cholangiography through the T-tube was performed without evidence of contrast passage into the duodenum (Fig. 1). The total and direct bilirubin had down trended to 1.5 and $1.3 \mathrm{mg} / \mathrm{dL}$ at that point.

A re-exploration of the bile duct was undertaken 30 days after the index operation. The previous Kocher incision was utilized. The common bile duct was found to be thickened and dilated. Re-entry into the common bile duct was performed utilizing the previous longitudinal incision, but it should be performed as close as possible to the duodenum, because if the sphincteroplasty is not feasible, a tension-free choledochoduodenostomy may be able to be performed. Using Randall forceps, a 5-mm stone embedded in the distal common bile duct was extracted with difficulty. Even after this, however, there was still no flow of contrast into the duodenum on cholangiography. At this point, it was decided to proceed with papillary exploration through a transduodenal approach. Bake's dilator was passed inside the bile duct toward the duodenum (this maneuver allows, through palpation of the dilator, for a more precise incision in relation to the ampulla to be made). An approximately 3-4 cm longitudinal incision was made in the second portion of the duodenum, along the antimesenteric border (Fig. 2). The dilator traversed the sphincter of Oddi with some difficulty due to stenosis secondary to fibrosis. Hemostasis should be achieved with careful electrocoagulation or an occasional 5/0 polyglycolic acid suture. Once adequate exposure of the ampulla was achieved, a papillotomy using a scalpel or Potts scissors against the Bakes' dilator was performed at the 10-11 o'clock position; all of these after placing stay sutures with 4-5/0 (polyproline or glycolic acid) along with the cardinal points of the papilla which are held with mosquito-Kellys to generate slight traction (Figs 2D to F). After

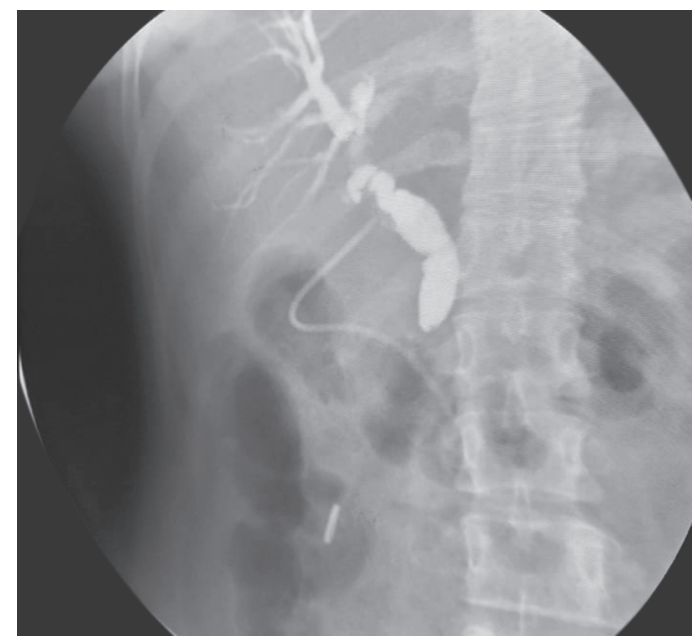

Fig. 1: T-tube cholangiography 30 days after the first bile duct exploration. A distal obstruction impeding the passage of contrast between the distal common bile duct and the duodenum is observed this, a 10-Fr catheter was able to be passed into the bile duct and bile flow into the duodenum was achieved (Fig. 2G). To perform a total Oddi sphincteroplasty, the incision should be almost 2 $\mathrm{cm}$ long, the same diameter as the common bile duct if there are impacted or residual stones. It is important to biopsy any suspicious areas and obtains a frozen section for intraoperative evaluation, if necessary. Once distal patency was confirmed, transverse closure of the duodenum has to be done in two layers by the usual method of inverting the mucosa with a Connell, Cushing, or continuous sero mucosal; then the serous layer should be closed with a Lembert suture or separate stitches with 4/0 polyglycolic acid (Fig. 2I). In this case, was using separate single layer 3-0 prolene sutures, which is feasible also when the diameter of the duodenum appears narrower than usual. Regarding the orientation of the closure, it is important to emphasize that if the stitches are of the appropriate thickness, there is no difference between the transverse or longitudinal closure of the duodenum and the relationship with the presentation of fistulas at this level. ${ }^{5-7}$

A 16-Fr latex " $T$ " tube was left in place in the common bile duct, and Jackson Pratt drain was left in Morrison's pouch.

The patient remained hospitalized for 72 hours. Liver function tests, including bilirubin, normalized. An oral diet was initiated 24 hours postoperatively and advanced on postoperative day 2. The patient was tolerating a full liquid diet through discharge. The T-tube output was quantified at an average of $200 \mathrm{~mL}$ daily. Cholangiography through the T-tube was done on postoperative day 9 at which point adequate passage of contrast into the duodenum was visualized. The output of the T-tube at that time was $<10 \mathrm{~mL}$ daily; as such, the drain was removed under cholangiography (Fig. 3).

\section{Discussion}

The presence of stones in any path of the biliary tree is called cholelithiasis. They appear as a consequence of the oversaturation of cholesterol in bile, inadequate levels of bile salts, and the decrease in contractility of the biliary epithelium due to the multifactorial effects of diet, hormones, and genetic predisposition. On the other hand, bile duct stones (choledocholithiasis) are most often the result of the migration of these stones from the gallbladder (cholecystolithiasis) to the main biliary trunk. ${ }^{1}$

Prospective data reveal that $10 \%$ of adults will develop symptomatic gallstones, and, of those between $10 \%$ and $20 \%$ will have concomitant choledocholithiasis. It is important to note that the presence of stones in the common bile duct is the leading cause of acute pancreatitis, which carries significant morbidity and mortality. ${ }^{2}$

The inflammatory process of the sphincter of Oddi, also known as stenosing papillitis, is overall rare but is three times more common in women between 30 years and 50 years old. Stenosing papillitis can be primary or secondary due to the presence of stones in the common bile duct; secondary makes up $10.8 \%$ of all stenosing papillitis. Macroscopically, in the acute state, the papilla can present with phlegmonous swelling, appearing red and edematous. The stenosis can cover just portions of the entire sphincteric apparatus, which encompasses the ampulla, common bile duct, and pancreas. ${ }^{3}$

The diagnostic method of choice for choledocholithiasis in our setting is magnetic resonance cholangiopancreatography (MRCP). However, it has been described that endoscopic ultrasound (EUS) is more sensitive. The limited availability of EUS in our environment makes it a difficult-to-access method. ${ }^{4}$ 

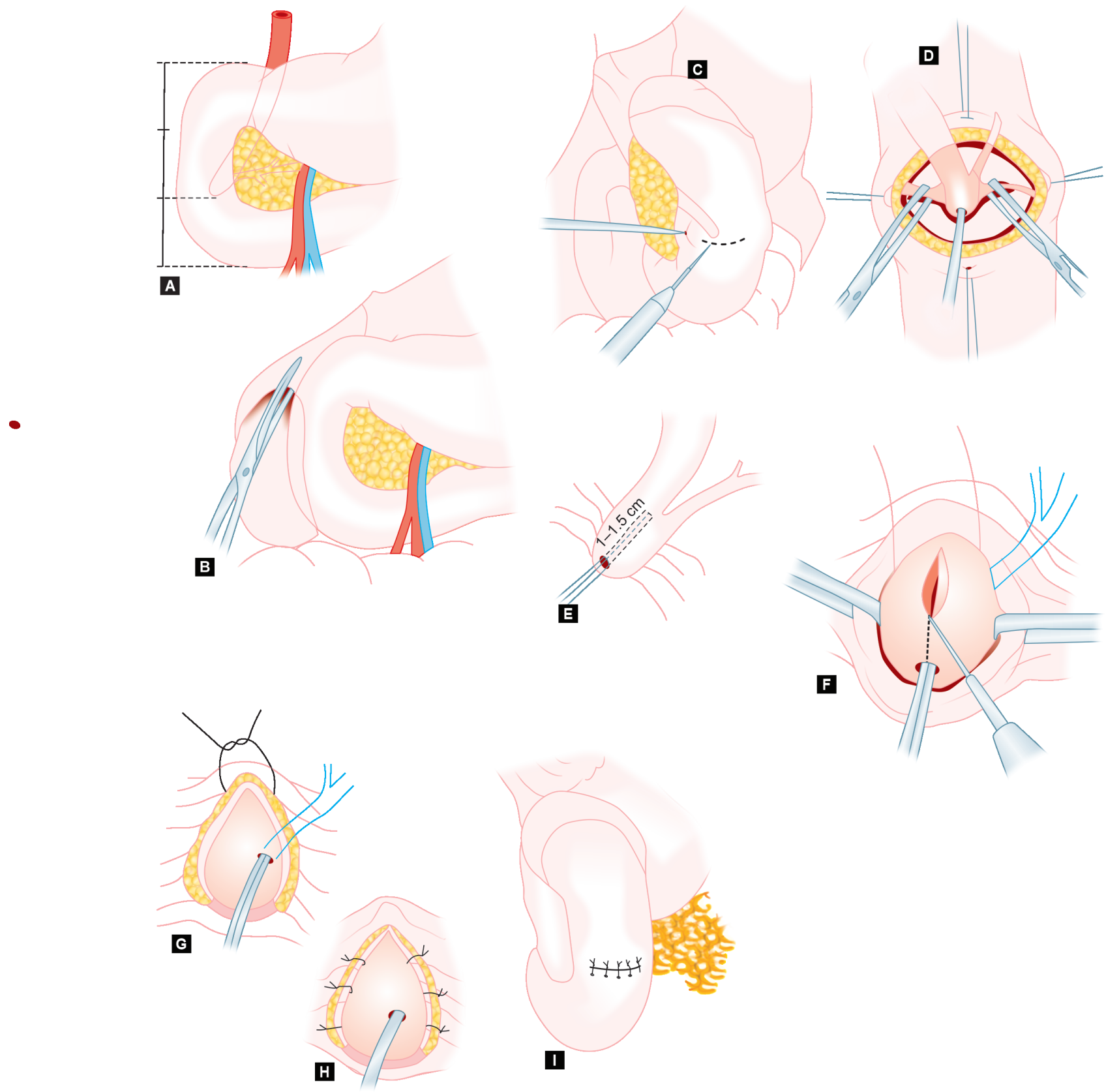

Figs 2A to I: Surgical technique "Transduodenal sphincteroplasty". The ampulla of Vater position in relation to the (A) second duodenal loop and (B) duodenal mobilization. Incision of the duodenum after the wall, opposite the ampulla of Vater, is exposed by (C) rotating the mobilized duodenum to the left; the papilla is exposed and (D) a grooved director is introduced into its proximal end. Following the guidance of the (E) grooved director, the (F) papilla is incised for 1-1.5 cm. After papillostomy, $(\mathrm{G})$ sinus probe has been introduced into the proximal end of the Wirsung duct. Suture of the medial aspect of papillotomy $(\mathrm{H})$ is completed. (I) Duodenal closure. Taken from: Miccini et al. ${ }^{10}$

Each year, choledocholithiasis causes biliary obstruction, cholangitis, and pancreatitis in a significant number of patients. The initial treatment, ERCP, is minimally invasive but is associated with adverse events in $6-15 \%$ of cases. Among the complications are post-ERCP pancreatitis with a frequency of $1.3-6.7 \%$, and technical failure occurring in $0.6-1 \%$ (as in the case of our patient) of cases. For these reasons, one must be familiar with alternatives such as laparoscopic or conventional bile duct exploration, transduodenal sphincterotomy, or even a choledochoduodenostomy. ${ }^{4}$
Although with the advent of new technology in laparoscopy, especially with the development of the choledochoscope, which can be introduced through the cystic duct or the common bile duct to directly visualize the extrahepatic and intrahepatic biliary tree, guaranteeing the complete elimination of stones, allowing for the inspection of the distal common bile duct, and detecting the presence of anomalies of the sphincter of Oddi, this tool is not available in our limited-resource hospital. ${ }^{6}$

For this reason, despite the development of modern endoscopic and laparoscopic techniques, open bile duct exploration continues 


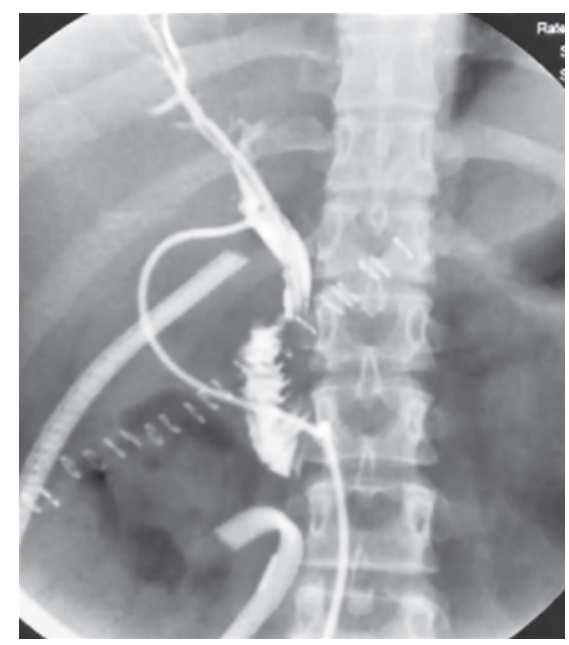

Fig. 3: T-tube cholangiography on day 9 after reoperation. Passage of contrast into the duodenum is visualized

to be a good treatment option at some hospitals in developing countries and for a certain subset of patients (previous surgery with dense adhesions, aberrant biliary ductal anatomy, failed ERCP). ${ }^{8}$

The transduodenal sphincterotomy is indicated for stones impacted at the ampulla of Vater, allowing an adequate visualization of the duodenal papilla, as well as its safe dilation and the concomitant extraction of stones. The surgical technique was described before in this case report. ${ }^{9}$

It is important to remark some points of postoperative care, to obtain a better recovery process for the patient. The nasogastric tube should be kept for approximately 2 days or until there is evidence of peristalsis with the passage of the flatus. The serum amylase level can be performed every 2 days or if there is a clinical picture of pancreatitis. Perioperative antibiotics should be maintained for 72 hours, except when the patient has concomitant cholangitis, in which case it should be continued for at least 7 days. A cholangiography should be performed on the seventh postoperative day. If the results are favorable, i.e., there is good contrast flow into the duodenum and in the absence of a fistula, the negative suction can be removed that day. After 14 days the T-tube drain can be removed. ${ }^{9}$

Complications: The most common and serious complication is the duodenal fistula, which can be even fatal if it is a high flow, requiring reoperations such as Billroth II gastrectomy with vagotomy and even Roux-en-Y hepaticojejunostomy. ${ }^{8-10}$

\section{Conclusion}

Transduodenal sphincteroplasty is a procedure that is rarely performed in the era of endoscopy. However, knowing the appropriate surgical technique for specific cases where this technique is necessary is crucial to obtain satisfactory results for the patient.

\section{References}

1. Takada T. Tokyo guidelines 2018: updated Tokyo guidelines for the management of acute cholangitis/acute cholecystitis. J Hepatobiliary Pancreat Sci 2018;25(1):1-2. DOI: 10.1002/jhbp.526.

2. Castillo Castellanos R, Sndoval Valenzuela E, González Hernández $M$, et al. Papilitis estenosante primaria. Salud en Tabasco [en linea] 2001;7(2):404-407. [fecha de Consulta 5 de Abril de 2021]. ISSN: 1405-2091. Disponible en: https://www.redalyc.org/articulo. oa?id=48707207.

3. Ceppa EP, Thomas KM, Schwartz PB. Advanced biliary procedures. The SAGES manual of biliary surgery. Cham: Springer; 2020. pp. 265-285.

4. Buxbaum JL, Abbas Fehmi SM, Sultan S, et al. ASGE guideline on the role of endoscopy in the evaluation and management of choledocholithiasis. Gastrointest Endosc 2019;89(6):1075-1105.e15. DOI: 10.1016/j.gie.2018.10.001.

5. Scott-Conner CEH, Chassin JL. Sphincteroplasty: surgical legacy technique. In: Scott-Conner C, ed. Chassin's operative strategy in general surgery. New York: Springer; 2014. DOI: 10.1007/978-1-46141393-6_82.

6. Gad EH, Zakaria H, Kamel Y, et al. Surgical (open and laparoscopic) management of large difficult CBD stones after different sessions of endoscopic failure: a retrospective cohort study. Ann Med Surg (Lond) 2019;43:52-63. DOI: 10.1016/j.amsu.2019.05.007.

7. Peel AL, Hermon-Taylor J, Ritchie HD. Technique of transduodenal exploration of the common bile duct. Duodenoscopic appearances after biliary sphincterotomy. Ann R Coll Surg Engl 1974;55(5): 236-244.

8. Manes G, Paspatis G, Aabakken L, et al. Endoscopic management of common bile duct stones: European Society of Gastrointestinal Endoscopy (ESGE) guideline. Endoscopy 2019;51(5):472-491. DOI: 10.1055/a-0862-0346.

9. Mesleh MG, Asbun HJ. Management of common bile duct injury. In: Asbun HJ, Shah MM, Ceppa EP, et al., ed. The SAGES manual of biliary surgery [Internet]. Cham: Springer International Publishing; 2020. [cited janvier 14, 2020] pp. 213-231. Available at: 10.1007/978-3-03013276-7_14.

10. Miccini $M$, Bonapasta $S$, Gregori $M$, et al. Indications and results for transduodenal sphincteroplasty in the era of endoscopic sphincterotomy. Am J Surg 2010;200(2):247-251. DOI: 10.1016/j. amjsurg.2009.08.048. 